\title{
Emergence of Space-Time from Topologically Homogeneous Causal Networks
}

\author{
Giacomo Mauro D'Ariano and Alessandro Tosini \\ QUIT Group, Dipartimento di Fisica "A. Volta", via Bassi 6, I-27100 Pavia, \\ Italy and Istituto Nazionale di Fisica Teorica e Nucleare, Sezione di Pavia.
}

\begin{abstract}
In this paper we study the emergence of Minkowski space-time from a causal network. Differently from previous approaches, we require the network to be topologically homogeneous, so that the metric is derived from pure event-counting. Emergence from events has an operational motivation in requiring that every physical quantity - including space-time - be defined through precise measurement procedures. Topological homogeneity is a requirement for having space-time metric emergent from the pure topology of causal connections, whereas physically homogeneity corresponds to the universality of the physical law. We analyze in detail the case of $1+1$ dimensions. If we consider the causal connections as an exchange of classical information, we can establish coordinate systems via an Einsteinian protocol, and this leads to a digital version of the Lorentz transformations. In a computational analogy, the foliation construction can be regarded as the synchronization with a global clock of the calls to independent subroutines (corresponding to the causally independent events) in a parallel distributed computation. Thus the Lorentz time-dilation emerges as an increased density of leaves within a single tic-tac of a clock, whereas space-contraction results from the corresponding decrease of density of events per leaf. The operational procedure of building up the coordinate system introduces an in-principle indistinguishability between neighboring events, resulting in a network that is coarse-grained, the thickness of the event being a function of the observer's clock. The illustrated simple classical construction can be extended to space dimension greater than one, with the price of anisotropy of the maximal speed, due to the Weyl-tiling problem. This issue is cured if the causal network is quantum, as e.g. in a quantum cellular automaton, and isotropy is recovered by quantum coherence via superposition of causal paths. We thus argue that in a causal network description of space-time, the quantum nature of the network is crucial.
\end{abstract}

\section{INTRODUCTION}

Our everyday way of looking at space-time as a stage for physical events conflicts with the requirement of defining all physical quantities - including space and timethrough precise measurement protocols. This means that we should more properly regard space-time as emerging from events, instead of pre-existing them. The operational definition of space-time is defined by the protocol that sets up the coordinate system. For example, in the Einstein protocol light pulses are sent back and forth between different locations: at the place where the signal has been originated, from the arrival time of the reflected signal one infers both the distance and the time of the remote event of signal-reflection. The protocol shows how space-time is indeed a coherent organization of inferences based on a causal structure for events. The clock itself is just a sequence of events - a light pulse bouncing between two mirrors. The closest are the mirrors, the more precise is the clock, and the more refined is the coordinate system.

The above reasoning shows that ultimately space and time are defined through pure event-counting, precisely counting tic-tacs of the observer's clock, and we are thus lead to regard space and time as emergent from the topology of the causal network of events. The events of the network do not need to be regarded as actual, but can be just potential, and the fabric of space-time is precisely the network of causal links between them.

The idea of deriving space-time from purely causal structures is not new. Raphael Sorkin started an inde- pendent research line in quantum gravity based on this idea more than two decades ago [1]. This was motivated by the potentialities of the approach residing in the natural discreteness of the causal network, which also provides a history-space for a path integral formulation [2, 3. The possibility of recovering the main features of the space-time manifold - topology, differentiable structure, and conformal metric - has been shown, starting from discrete sets of points endowed with a causal partial ordering [1. Along these lines, in an operational context, Lucien Hardy has also formulated a causaloid approach [5], which considers the possibility of a dynamical treatment of the causal links.

In the causal-set approach of Sorkin events are randomly scattered in order to avoid occurrence of their sparseness in the boosted frames that would lead to violation of Lorentz invariance. Clearly the randomness of Sorkin's events should not be regarded in terms of their location on a background, otherwise we contradict the same idea of space-time emergence. We then need to consider randomnes at the pure topological level, and this means having random causal connections. However, regarding the causal connections as an irreducible description of the physical law, a random topology would then corresponds to having a random physical law at the most microscopic level (the Planck scale), and one may argue that a "random law" would contradict the same notion of law. Instead, the randomnes should results from the law itself, e.g. in a quantum cellular automaton, where randomness comes from quantum nature of the network. The universality of the physical law thus lead us to take the causal network as topologically ho- 
mogeneous. Topological homogeneity has then the added bonus that metric simply emerges from the pure topology by just counting events along the network. It is obvious that the discreteness of the network will lead to violation of Lorenz covariance (and the other space symmetries) at the Planck scale level: however, one must have a theory where covariance is restored in the large scale limit-the Fermi scale-corresponding to counting huge numbers of events.

With the above motivations, in this paper we analyze the mechanism of emergence of space-time from the pure homogeneous topology in $1+1$ dimensions. We present a digital version of the Lorentz transformations, along with the corresponding digital-analog conversion rule. Upon considering the causal connections as exchanges of classical information, we can establish coordinate systems via an Einsteinian protocol, leading to a digital version of the Lorentz transformations. In a computational analogy first noticed by Leslie Lamport [6], the foliation construction can be regarded as the synchronization protocol with a global clock of the calls to independent subroutines (the causally independent events) in a parallel distributed computation. The boosts are determined by the relative lengths of the tic and tac of the clock, and the Lorentz time-dilation corresponds to an increased number of leaves within a clock tic-tac, whereas space-contraction results from the corresponding decreased density of events per leaf, as first noticed in Ref. [7].

We will see that the operational procedure of building up the coordinate system introduces an in-principle indistinguishability between neighboring events, resulting in a network that is coarse-grained, the thickness of the event being a function of the observer's clock. The digital version of the Lorentz transformation is an integer relation which differs from the usual analog transformation by a multiplicative real constant corresponding exactly to the event thickness. The composition rule for velocities is independent on such constant, and is the same in both the analog and the digital versions. Preliminary results of the present work were already presented in Ref. [8].

The illustrated simple classical construction can be extended to space dimension greater than one, but at the price of anisotropy of the maximal speed, due to the Weyl-tiling problem. This issue is cured if the causal network is quantum, as e.g. in a quantum cellular automaton, and isotropy is recovered with quantum coherence, corresponding to superposition of causal paths. We will thus argue that in a causal network description of spacetime, the quantum nature of the network is crucial.

\section{SETTING UP THE DIGITAL COORDINATE SYSTEM}

The first problem to address is which specific lattice should be adopted for the causal network. In our convention the causal arrow is directed from the bottom to the top of the network. The dimension of the emerging spacetime corresponds to the graph-dimension of the network, which is the dimension of the embedding manifold such that all links can be taken as segments of straight line with the same length. We will require that the lattice be pure topology (namely with all events equivalent), corresponding to a locally homogeneous spacetime, and with no redundant links. It is then easy to see that in $1+1$ dimensions there are only three possible lattices: the square, the triangular, and the honeycomb ones. The honeycomb-lattice has two inequivalent types of events (having one input and two output links and viceversa), and the corresponding "undressed" topology - where each couple of connected inequivalent events are merged into a single event-reduces to the square lattice. The triangular-lattice, on the other hand, has redundant causal links (the middle vertical ones). We are thus left with the square-lattice.
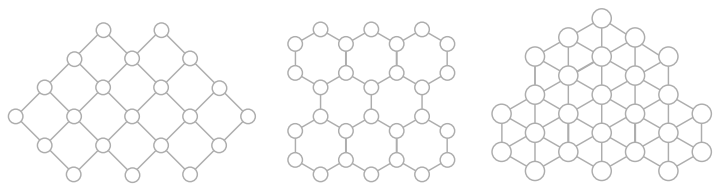

FIG. 1: The three possible homogeneous topologies for causal networks in $1+1$ dimensions.

We always assume the network links as oriented according to the causal arrow. In the square-lattice network there are thus two types of link: toward the right and toward the left - shortly $r$-link and $l$-link. Two events are in the same position (for some boosted reference frame) if they are connected by a path made with a sequence of $r$-links followed by a sequence of $l$-links. When the two sequences contain the same number of links the reference is at rest.

A clock is a sequence of causally connected events periodically oscillating between two positions. For an Einstein clock the oscillation (tic-tac) is exactly the same couple of sequences of $l$ - and $r$-links identifying events in the same position. The precision of the clock, namely the minimum amount of time that it can measure, is the number of links of a complete tic-tac. The tic-tac is indivisible, namely the sole tic (or tac) is not a complete measured time interval, since it involves two different positions.

In the following we will call light signals those sequences of events that are connected only by $r$-links or by $l$-links, namely making segments at 45 degrees with the horizontal in the network. Their "speed" is equal to "one event-per-step", and is the maximum speed allowed by the causality of the network, since connecting events along a line making an angle smaller than $45^{\circ}$ with the horizontal would require to follow some causal connections in the backward direction from the output to the input. In this way in a homogeneous causal network suffices to guarantee a bound for the speed of information flow. 


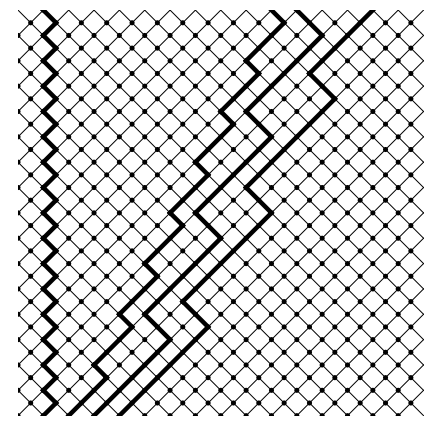

FIG. 2: Different clocks on the causal network. The tic-tac of the clock is represented by the two numbers $\alpha$ and $\beta$ (see text). From the left to the right we have the rest-frame clock, clock corresponding to $\alpha=\beta=1$, and boosted-frames for $\alpha=3, \beta=1, \alpha=6, \beta=2$, and $\alpha=7, \beta=2$, respectively, corresponding to digital speed $v=\frac{1}{3}, v=\frac{1}{3}$ and $v=\frac{2}{7}$, respectively. The case $\alpha=6, \beta=2$ has doubled imprecision, compared to the case $\alpha=3, \beta=1$.

In the following we will take the clock tic-tac made with $\alpha r$-links followed by $\beta$ l-links (see Fig, 2). Any clock allows to introduce a reference frame $\mathfrak{R}$ which is just a foliation of the network built up using the Einstein protocol. From the start of the clock tic-tac a light signal is sent to an event in a different position and then received back at the clock. The intermediate time between the sending and the receiving event is taken as synchronous with the event at the turning point, and the number of tic-tacs divided by two is taken as the distance from the turning point and the clock conventionally located at the beginning of the tic-tac. In this way we build the foliation corresponding to a given clock. A set of synchronous events identifies a leaf of the foliation. In Fig. 3 the Einstein protocol is illustrated in two particular reference frames. The figure on the left corresponds to the rest-frame, with the blue lines depicting the coordinate system established using the clock with $\alpha=\beta=1$ (see Fig. 2). The green lines represent light signals bouncing between the clock and four particular events in the network. These events are synchronous, since the intermediate time between the sending and the receiving event on the clock is the same for all of them. They lie on the same leaf of the foliation, but at different position, $0,1,4,7$, respectively: the spatial coordinate is obtained by counting the tic-tacs between the the sending and the receiving event divided by two. The right figure represents a boosted frame for $\alpha=3, \beta=1$, built up using the same protocol as in the left figure.

Due to indivisibility of the tic-tac, we see that there are indiscernible events, for which the synchronisation occurs in the middle of the tic-tac. We are thus led to identify events, and merge them into thicker coarse-grained events. This is done as follows. We identify the events along the tic and those along the tac so that the tictac is always regarded as the bouncing between two next neighbour events. Then we merge events into minimal sets so that the topology is left the invariant (see figures

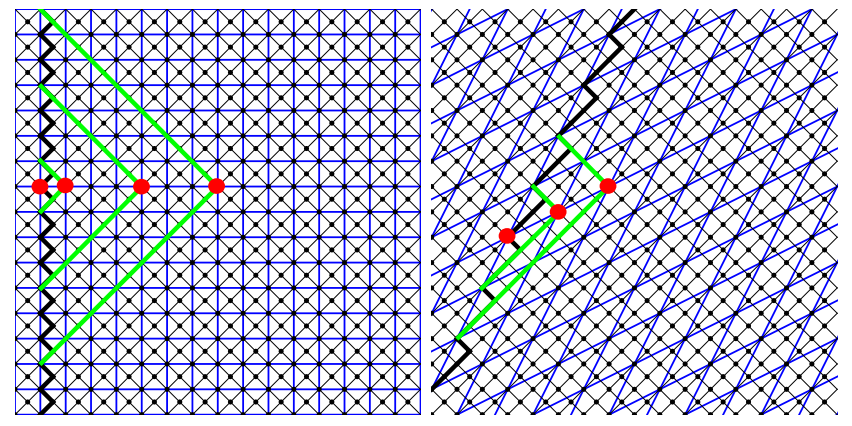

FIG. 3: Illustration of the Einstein protocol for establishing a reference frame. Left figure: Rest-frame. The blue lines represent the reference frame established using the clock with $\alpha=\beta=1$ (see Fig. 2). The green lines represent light signals bouncing between the clock and four particular events in the network. These events are synchronous, since the intermediate time between the sending and the receiving event on the clock is the same for all of them. They lie on the same leaf of the foliation, but at different position, $0,1,4,7$, respectively: the spatial coordinate is obtained by counting the tic-tacs between the the sending and the receiving event $(0,2,8,14$, respectively) divided by two. Right figure: Boosted reference frame (blue lines) for $\alpha=3, \beta=1$, built up using the same protocol as in the felt figure.

4 and 5). We can distinguish between two different kinds of coarse-graining: one due to the boosting (in yellow in the figures), and one due to intrinsic imprecision of the clock (in gray). The difference between the two is clarified in Fig. 5. In the top figure events along the tic and events along the tac are identified in the boosted frame. Then events are merged into minimal sets (in yellow) so that the topology is left invariant (the merged events are again events of a square-lattice network). In the central figure the coarse graining associated to the intrinsic imprecision is added in gray, and finally, in the bottom figure the circuit is stretched so to have all synchronous events on horizontal lines, and events located in the same position on vertical lines. Notice that in the special case of the rest-frame, see Fig. \$, the coarse-graining is just due to the intrinsic imprecision of the clock.

\section{THE DIGITAL LORENTZ TRANSFORMATIONS}

The velocity of the boosted frame can be easily written in terms of the $\alpha$ and $\beta$ of the tic-tac of the clock, by simply evaluating the ratio of the distances in space and time between the two ending points of the tic-tac, namely

$$
v=\frac{\alpha-\beta}{\alpha+\beta} .
$$

Thanks to invariance of topology under the boost coarsegraining, the above identity holds also for the motion relative to any boosted frame, whence, upon defining with $\alpha_{12}=\alpha_{2} / \alpha_{1} \in \mathbb{Q}(\mathbb{Q}$ denoting rational numbers $)$ and 


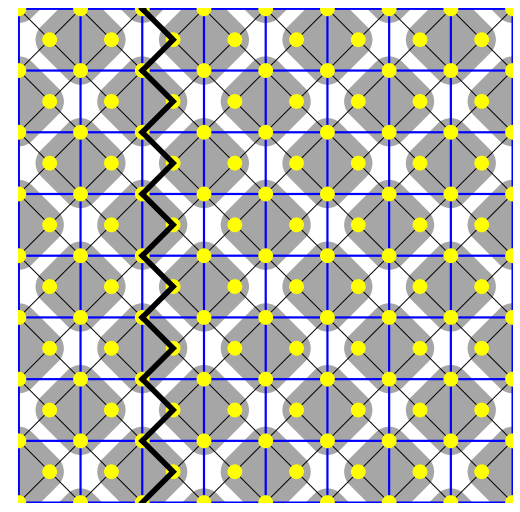

FIG. 4: Coarse-graining in the rest-frame. The coarsegraining due to boosts is trivial (depicted in yellow, containing just one event), whereas the coarse-graining associated to the intrinsic imprecision of the clock is not trivial (depicted in gray, containing four events).

$\beta_{12}=\beta_{2} / \beta_{1} \in \mathbb{Q}$ for frames $\Re_{1}$ and $\Re_{2}$, and by $v_{12} \in \mathbb{Q}$ the relative velocity of frame $\Re_{2}$ with respect to frame $\Re_{1}$, one has

$$
v_{12}=\frac{\alpha_{12}-\beta_{12}}{\alpha_{12}+\beta_{12}}
$$

Now, by using the trivial identities $\alpha_{13}=\alpha_{12} \alpha_{23}$ and $\beta_{13}=\beta_{12} \beta_{23}$ one has

$$
v_{13}=\frac{\alpha^{12} \alpha^{23}-\beta^{12} \beta^{23}}{\alpha^{12} \alpha^{23}+\beta^{12} \beta^{23}},
$$

which by simple algebraic manipulations immediatley gives

$$
v_{13}=\frac{\left(\frac{\alpha^{12}-\beta^{12}}{\alpha^{12}+\beta^{12}}\right)+\left(\frac{\alpha^{23}-\beta^{23}}{\alpha^{23}+\beta^{23}}\right)}{1+\left(\frac{\alpha^{12}-\beta^{12}}{\alpha^{12}+\beta^{12}}\right)\left(\frac{\alpha^{23}-\beta^{23}}{\alpha^{23}+\beta^{23}}\right)}=\frac{v_{12}+v_{23}}{1+v_{12} v_{23}} .
$$

The last identity is the composition rule of parallel velocities (the only possibility in $1+1$ dimension) in special relativity.

Now we use the Einstein protocol to construct the boosted coordinated system with respect to the restframe along with the relative coordinate systems between any couple of boosted frames. We will now see that the coordinates of an event transform from the frame $\Re_{2}$ to the frame $\mathfrak{R}_{1}$ as follows

$$
\begin{aligned}
& s_{1}=\frac{1}{2}\left(\alpha_{12}+\beta_{12}\right) s_{2}+\frac{1}{2}\left(\alpha_{12}-\beta_{12}\right) t_{2}, \\
& t_{1}=\frac{1}{2}\left(\alpha_{12}+\beta_{12}\right) t_{2}+\frac{1}{2}\left(\alpha_{12}-\beta_{12}\right) s_{2} .
\end{aligned}
$$

In fact, from a simple inspection of Figure 6 one can check Eqs. (5) and (6) with the frame $\Re_{1}$ as the rest frame. In the left figure the reference frame $\Re_{1}$ with $\alpha_{1}=\beta_{1}=1$ is represented by the tyny network in black, whereas the coarser network in blue represents the boosted reference frame $\Re_{2}$ with $\alpha_{2}=4, \beta_{2}=2$. According to Eq. (2) the

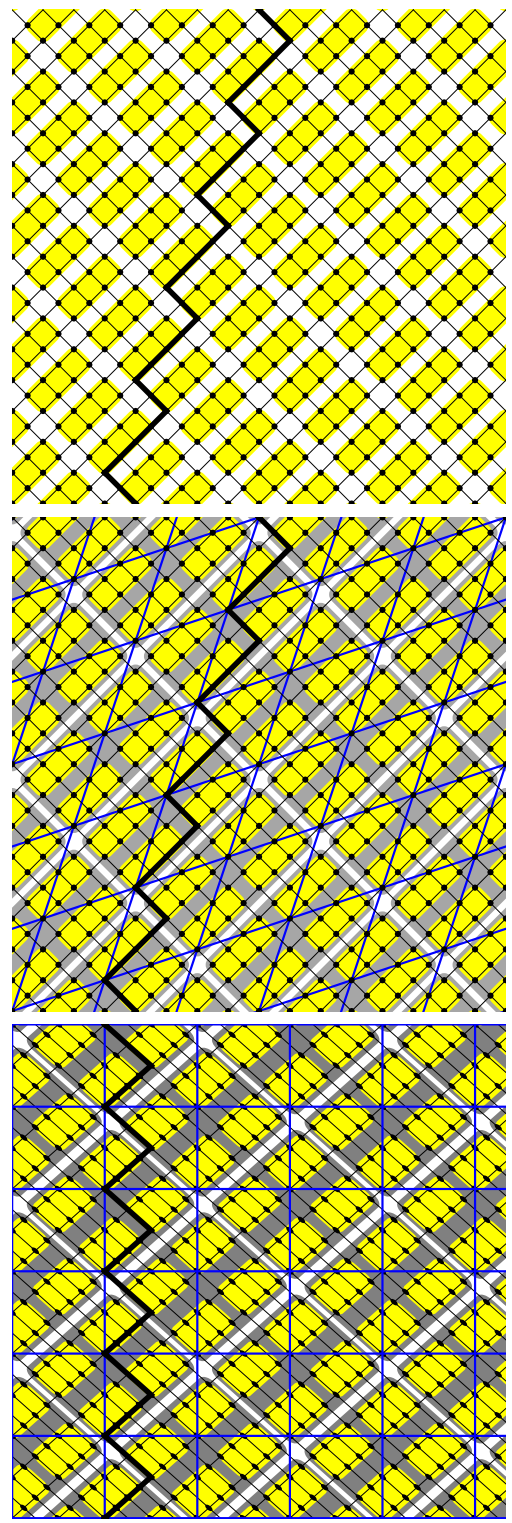

FIG. 5: Illustration of the coarse-graining procedure due to boost (in yellow) and to intrinsic imprecision of the clock (in gray). Top figure: events along the tic and events along the tac are identified in the boosted frame. Then events are merged into minimal sets (in yellow) so that the topology is left invariant (the merged events are again events of a squarelattice network). Center figure: the coarse graining associated to the intrinsic imprecision is added in gray. Bottom figure: the circuit is stretched so to have all synchronous events on horizontal lines, and events located in the same position on vertical lines.

relative velocity of $\Re_{2}$ with respect to $\Re_{1}$ is $v_{12}=1 / 3$. In order to connect the coordinate systems in the two frames we have chosen the same origin $(0,0)$ on both $\Re_{2}$ and $\Re_{1}$. The generic event on $\Re_{2} \cap \Re_{1}$ has coordinates $\left(s_{2}, t_{2}\right)=(3,2)$ and $\left(s_{1}, t_{1}\right)=(10,9)$ in the two frames, respectively. In the figure on the right one can see that a spatial step in $\mathfrak{R}_{2}$ corresponds to $\left(\alpha_{12}+\alpha_{12}\right) / 2$ space 


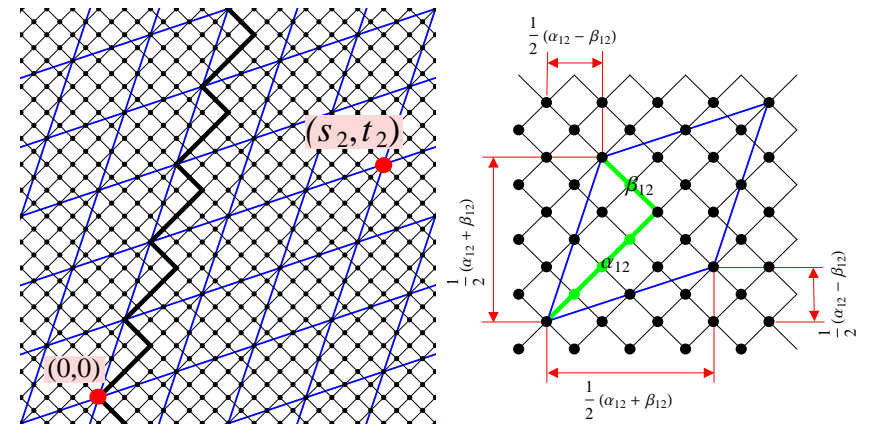

FIG. 6: Illustration of the derivation of Eqs. (5) and (6), leading to the digital version of the Lorentz transformations (7) and (\$). Left figure: the reference frame $\mathfrak{R}_{1}$ with $\alpha_{1}=\beta_{1}=1$ is represented by the tyny network in black, whereas the coarser network in blue represents the boosted reference frame $\mathfrak{R}_{2}$ with $\alpha_{2}=4, \beta_{2}=2$. According to Eq. (2) the relative velocity of $\mathfrak{R}_{2}$ with respect to $\mathfrak{R}_{1}$ is $v_{12}=1 / 3$. In order to connect the coordinate systems in the two frames we have chosen the same origin $(0,0)$ on both $\mathfrak{R}_{2}$ and $\mathfrak{R}_{1}$. The generic event on $\mathfrak{R}_{2}$ has coordinates $\left(s_{2}, t_{2}\right)=(3,2)$ and $\left(s_{1}, t_{1}\right)=(10,9)$ in the two frames, respectively. Right figure: a spatial step in $\mathfrak{R}_{2}$ corresponds to $\left(\alpha_{12}+\alpha_{12}\right) / 2$ space and $\left(\alpha_{12}-\alpha_{12}\right) / 2$ time steps in $\mathfrak{R}_{1}$. In the same way to a time step in $\mathfrak{R}_{2}$ corresponds to $\left(\alpha_{12}-\alpha_{12}\right) / 2$ space and $\left(\alpha_{12}+\alpha_{12}\right) / 2$ time steps in $\Re_{1}$. This correspondence allows to determine the coordinates of a given event in the frame $\left(s_{1}, t_{1}\right)$ in terms of its coordinates $\left(s_{2}, t_{2}\right)$ in the frame $\mathfrak{R}_{2}$. The resulting transformations are in Eqs. (5) and (6).

and $\left(\alpha_{12}-\alpha_{12}\right) / 2$ time steps in $\Re_{1}$. In the same way a time step in $\Re_{2}$ corresponds to $\left(\alpha_{12}-\alpha_{12}\right) / 2$ space and $\left(\alpha_{12}+\alpha_{12}\right) / 2$ time steps in $\Re_{1}$. This correspondence allows to determine the coordinates $\left(s_{1}, t_{1}\right)$ of a given event in the frame $\Re_{1}$ in terms of its coordinates $\left(s_{2}, t_{2}\right)$ in the frame $\mathfrak{R}_{2}$. The resulting transformations are in Eqs. (5) and (6). Invariance of topology with boost, guarantees that they also hold between any couple of boosted frames. By elementary manipulation Eqs. (5), (6) can be written in the more customary way

$$
\begin{aligned}
& s_{1}=\frac{1}{2}\left(\alpha_{12}+\beta_{12}\right)\left(s_{2}+v_{12} t_{2}\right), \\
& t_{1}=\frac{1}{2}\left(\alpha_{12}+\beta_{12}\right)\left(t_{2}+v_{12} s_{2}\right) .
\end{aligned}
$$

Upon defining the following constant depending on the clocks of the two frames

$$
\chi_{12}:=\sqrt{\alpha_{12} \beta_{12}}
$$

and using the identity

$$
\frac{1}{2}\left(\alpha_{12}+\beta_{12}\right)=\frac{\chi_{12}}{\sqrt{1-v_{12}^{2}}},
$$

we obtain the digital Lorentz transformations

$$
s_{1}=\chi_{12} \frac{s_{2}+v_{12} t_{2}}{\sqrt{1-v_{12}^{2}}}, \quad t_{1}=\chi_{12} \frac{t_{2}+v_{12} s_{2}}{\sqrt{1-v_{12}^{2}}}
$$
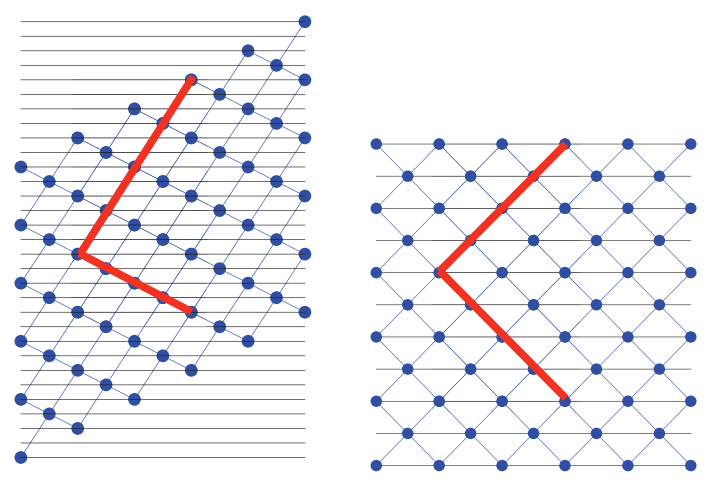

FIG. 7: The mechanism for the digital Lorentz time-dilation and space-contraction given in Ref. [7], here for a boost with $v=\frac{1}{2}$, corresponding to a digital time-dilation by a factor 2 (analog factor $2 / \sqrt{3}$ ) and space-contraction by a factor $1 / 2$ (compare with the same factors in Eqs. (11)).

Eqs. (11) differ from the usual analog Lorentz transformations by the multiplicative factor $\chi_{12}$, which is logically required to make the transformations rational, compensating the irrationality of the boost factor $\sqrt{1-v_{12}^{2}}$. The digital-analog conversion is thus just a rescaling of both space and time coordinates by the factor $(\alpha \beta)^{\frac{1}{2}}$ depending on the boost, which is exactly the square-root of the volume of the coarse-grained event measured as the number of rest-frame events that it contains. Such event volume also affects the Lorentz space-contraction and time-dilation factor, which in the digital case is given by $\frac{1}{2}\left(\alpha_{12}+\beta_{12}\right)$, whereas in the analog case is rescaled by the ratio of event volumes, leading to $\frac{1}{2 \sqrt{\alpha_{12} \beta_{12}}}\left(\alpha_{12}+\beta_{12}\right)$. Thus, for example, for $\alpha_{12}=1$ and $\beta_{12}=3$ corresponding to $v_{12}=1 / 2$ the digital factor is 2 whereas the analog one is $2 / \sqrt{3}$. The digital factor agrees with that of the Lorentz time-dilation and space-contraction mechanism of Ref. 7, given in terms of increased density of leaves and corresponding decreased density of events per leaf, as illustrated in Fig. 7 .

\section{CONCLUSIONS AND DISCUSSION}

We have analyzed the mechanism of emergence of space-time from homogeneous topology in $1+1$ dimensions, deriving the digital version of the Lorentz transformations along with the corresponding digital-analog conversion rule. The homogeneity of topology physically represents the universality of the physical law (it is worth mentioning that such law is stripped of the conventionality of space and time homogeneity: see e.g. Ref. [9]). We have built the digital coordinate system using the Einstein's protocol, with signals sent back-and-forth to events from an observer's clock. We found that the procedure introduces an in-principle indistinguishability between neighbouring events, due to the limited precision of 
the clock, resulting in a network that is coarse-grained, with the event thickness also depending on the boost. The digital version of the Lorentz transformation is an integer relation which differs from the usual analog transformation by a multiplicative real constant corresponding to the event thickness.

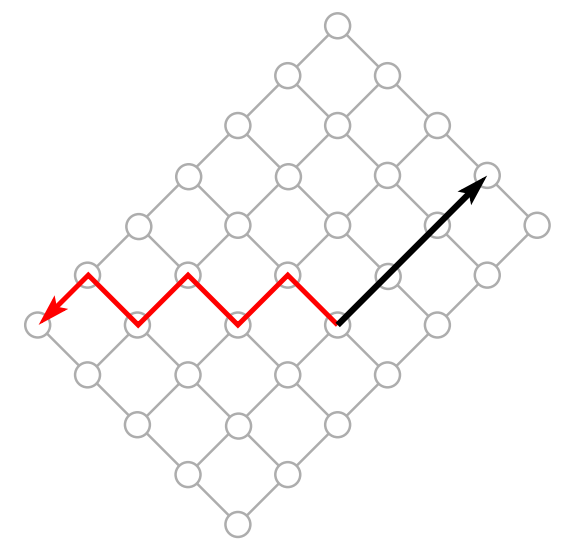

FIG. 8: Square $(1+2)$-dimensional computational network: view of a leaf in the rest frame. Information must zig-zag to flow at the maximal speed in diagonal direction. This leads to a slow-down of a $\sqrt{2}$ factor of the analog speed compared to cubic axis direction.

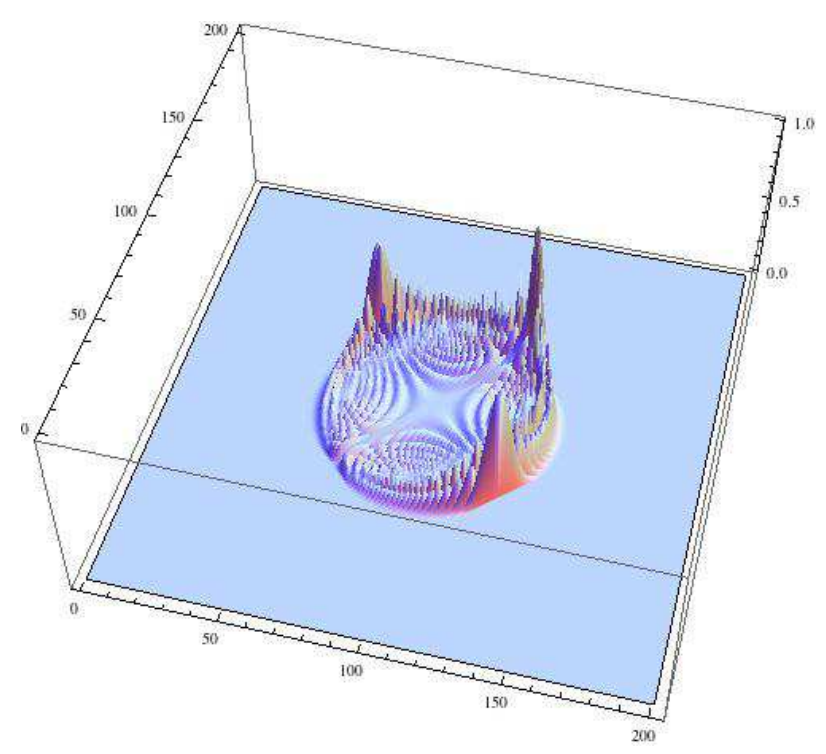

FIG. 9: Evolution for 60 steps of the probability distribution of finding a particle or antiparticle in a two-dimensional quantum Weyl automaton of the kind of Bialynicki-Birula 12 on a square-lattice. One can see how the propagation speed is isotropic after few steps.
The present purely classical cinematical construction does not straightforwardly extend from one dimension to larger dimensions, due to the Weyl-tiling issue, namely that continuum geometry cannot simply emerge from counting sites on a discrete lattice, since e.g. in a square tiling one counts the same number of tiles along a side and along the diagonal of a square [10]. Thus, for example, as shown in Fig. 8, in a causal network shaped as a square-lattice the fastest speed would be along the cubic axes, whereas along diagonals information should zig-zag, resulting in a slowdown by a factor $\sqrt{2}$ (or even $\sqrt{3}$ in three dimensions). Indeed, a general theorem of Tobias Fritz [11] shows that the polytope of points that can be reached in no more than $N$ links in a periodic graph does not approach a circle for large $N$. Since the polytope has necessarily distinguished directions, this means that there is no periodic graph for which this velocity set is isotropic. This result represents a no-go theorem for the emergence of an isotropic space from a discrete homogeneous causal network representing a classical information flow.

The situation, however, is completely different if one considers the possibility that information can flow in a superposition of paths, along the network, as in a quantum cellular automaton, corresponding to a homogeneous quantum computational network. In Fig. 9 a concrete example of evolution is given for a two-dimensional quantum Weyl automaton of the kind of Bialynicki-Birula 12] on a square-lattice. One can see that the maximum propagation speed is isotropic after just few steps. In a similar way full Lorentz covariance is expected to be restored in the same limit of infinitely many events-a kind of thermodynamic limit bringing the automaton to the Fermi scale.

\section{Acknowledgements}

GMD acknowledges interesting discussions with Raphael Sorkin, Seth Lloyd, and Tobias Fritz. This work has been partially supported by PRIN 2008.
[1] L. Bombelli, J. H. Lee, D. Meyer, and R. Sorkin, Phys. Rev. Lett 59, 521 (1987).

[2] F. Markopoulou, gr-qc/0210086 (2002).
[3] J. Henson, in Approaches to Quantum Gravity: Towards a New Understanding of Space and Time, Ed. D. Oriti (Cambridge University Press, Cambridge UK, 2006) (also 
gr-qc/0601121).

[4] S. Surya, Theor. Comp. Sc. 405188 (2008).

[5] L. Hardy, J. Phys. A 403081 (2007).

[6] L. Lamport, Comm. ACM, 21558 (1978).

[7] G. M. D'Ariano, in CP1232 Quantum Theory: Reconsideration of Foundations, 5 ed. by A. Y. Khrennikov, (AIP, Melville, New York, 2010), pg. 3 (also arXiv: 1001.1088).

[8] G. M. D'Ariano and A. Tosini, arXiv:1008.4805 (2010)
[9] D. Malament, Noûs 11293 (1977).

[10] H. Weyl, Philosophy of Mathematics and Natural Sciences, Princeton University

[11] T. Fritz, Velocity Polytopes of Periodic Graphs, draft (2011). Press, (Princeton 1949).

[12] I. Bialynicki-Birula, Phys. Rev. D 496920 (1994). 